\title{
Organogel formulations for the cleaning of easel paintings
}

\author{
Piero Baglioni ${ }^{1}$ - Nicole Bonelli ${ }^{1}$ - David Chelazzi ${ }^{1} \cdot$ Aurelia Chevalier $^{2}$. \\ Luigi Dei $^{1} \cdot$ Joana Domingues $^{1} \cdot$ Emiliano Fratini $^{1} \cdot$ Rodorico Giorgi $^{1}$ • \\ Morgane Martin ${ }^{2}$
}

Received: 31 March 2015/ Accepted: 14 July 2015/Published online: 31 July 2015

(C) Springer-Verlag Berlin Heidelberg 2015

\begin{abstract}
Gels are particularly useful for the cleaning of works of art, as they allow the controlled delivery of cleaning fluids on solvent-sensitive substrates such as easel paintings. Owing to the presence of covalent cross-links between the polymer chains, chemical gels exhibit mechanical properties that allow their easy handling and their residue-free removal from artistic surfaces after the cleaning intervention. Organogels based on the crosslinking of methyl methacrylate (MMA) can be prepared as loaded with solvents for the controlled removal of unwanted layers from the surface of canvas paintings. Here, we propose MMA-based organogels obtained by solubilizing MMA in pure organic solvents (e.g., ethyl acetate, butyl acetate and ketones) and using a dimethacrylate cross-linker. The uptake/release behavior of the gels has been investigated, and their mesoporosity has been characterized through small-angle X-ray scattering. Finally, the gels have been used for the removal of historical varnishes from canvas painting samples, checking the absence of gel residues with attenuated total reflectance Fourier transform infrared spectroscopy (ATR-FTIR).
\end{abstract}

Piero Baglioni

baglioni@csgi.unifi.it

1 Department of Chemistry Ugo Schiff and CSGI, University of Florence, Via della Lastruccia 3, 50019 Sesto Fiorentino, Florence, Italy

2 Atelier Aurelia Chevalier, Rue Saint Jacques 289, 75005 Paris, France

\section{Introduction}

The cleaning of easel paintings is a frequent task in restoration interventions, yet it poses challenges to conservators when it comes to achieving a controlled and selective removal of unwanted layers without affecting original components. Being the cleaning intervention irreversible, it needs to be carried out in a gradual way, for instance, aged varnishes are usually thinned layer-by-layer, rather than completely removed in a single step. Typically, materials that must be removed either from the surface or from the backside of easel paintings comprise deposits of soiling and grime, aged adhesives, varnishes or coatings. In fact, synthetic and natural polymer coatings can undergo yellowing and cracking, which affects the readability of the painted surface, while several adhesives (e.g., polyvinyl acetatebased products) can develop acidity that is detrimental to the support of the paintings (canvas fibers, wood) [1-4].

Traditionally, unwanted layers are removed coupling mechanical action with solubilization, the latter often obtained using organic solvents [5]. However, this operation involves several issues. First, the diffusion of free solvents through works of art can produce the swelling or solubilization of sensitive original components, such as pigments, dyes and binders. Moreover, any solubilized soiling, varnishes and adhesives can be re-transported by solvents within the pores of the artifact, which means that part of the unwanted layers is simply moved deeper within the painting, rather than actually removed from it. Finally, most organic solvents commonly used in restoration are volatile and toxic (to different degrees), hence potentially harmful both to operators and to the environment.

These issues can be addressed by confining the solvents within matrices that release them in a controlled way onto the artistic surface. To that end, gels represent ideal tools 
provided they exhibit some key features, especially in terms of retentiveness and mechanical behavior. Retentiveness means that the gel matrix releases the uploaded fluids at controlled rate, avoiding excessive wetting and uncontrolled diffusion of the solvents through the artifact's layers. As a result, retentive gels enable the gradual swelling, detachment or solubilization of unwanted surface layers while preserving the original components of the artifacts. In some cases, solubilized matter (e.g., dirt, aged varnishes) can migrate inside the gel, limiting transportation within the pores of the painting [6]. Good mechanical properties (e.g., high viscoelasticity) allow the easy handling of the gels and their complete removal from the surface of the painting after the cleaning intervention [7]. Moreover, the confinement of solvents reduces their volatility, and their ecotoxicological impact is decreased accordingly.

In fact, formulations with high retentiveness and optimal mechanical behavior have been targeted throughout the last decade to improve on traditional solvent thickeners based on cellulose ethers or polyacrylic acid, which are physical "gel-like" networks where the links between polymer chains are secondary bonds (hydrophobic, electrostatic, van der Waals interactions, hydrogen bonds). These polymer networks (typically labeled as "smooth" by conservators and conservation scientists) are prone to leaving residues that are hard to remove without using clearing solvents [810]. Alternatively, chemical gels (i.e., networks built on covalent cross-links between polymer chains) have been proposed as containers that can be loaded with cleaning fluids and applied on the surface of paintings [11, 12]. The network of covalent cross-links grants optimal mechanical properties, allowing the simple and residue-free removal of the gels after their application. In fact, in the conservation practice, chemical gels are among those defined as "rigid gels," owing to their mechanical behavior as opposed to that of "smooth" networks.

Recently, chemical hydrogels based on semiinterpenetrating networks of poly(2-hydroxymethyl methacrylate) (pHEMA) and poly(vinylpyrrolidone) (PVP) have been proposed for the cleaning of artifacts, as they combine the mechanical strength of pHEMA and the hydrophilic character of PVP. As a result, they can be loaded mainly with aqueous formulations or even with some polar solvents (e.g., methanol, ethanol, benzyl alcohol), and effectively applied for the removal of hydrophilic soiling and adhesives from canvas paintings [13].

Besides hydrogels, chemical "organogels" can also be considered, i.e., chemical networks where the confined liquid phase is composed of organic solvents, in particular exhibiting lower polarity as compared to those loaded in hydrogels. This makes organogels complementary to hydrogels as cleaning tools and expands further the applicability of chemical gels for the thinning and removal of natural or synthetic varnishes, coatings and adhesives. In the present study, chemical organogels based on methyl methacrylate (MMA) were prepared (by free radical copolymerization) as loaded with four organic solvents and applied for the first time on the surface of canvas painting samples. The preparation of chemical organogels through the polymerization of MMA and a cross-linker (usually a divinyl-group molecule) in a solvent solution (e.g., toluene) has been reported in the literature, for instance, for studying the diffusion of polymer chains in gels [14], for monitoring the swelling of gels through fast transient fluorescence [15] and for making optical lenses [16].

Here, MMA was solubilized in different pure organic solvents, namely methyl ethyl ketone (MEK), cyclohexanone (cyclo), ethyl acetate (EA) and butyl acetate (BA). These solvents were selected because they exhibit average polarity (e.g., as defined by their Teas solubility parameters), which makes them versatile solvents capable of dissolving or swelling a wide range of natural and synthetic resins that conservators frequently need to remove from works of art. Moreover, this set of solvents covers a range of different volatilities (in terms of boiling point, flash point and vapor pressure), which is advantageous in applications where the evaporation rate of solvents needs to match both practical and safety requirements. Therefore, it was important to see how these different solvents behaved when confined in PMMA gels and used for the cleaning of painting samples.

In order to obtain gel systems with the desired characteristics, some parameters were tuned, i.e., the amount of cross-linker, the type of solvent and the monomer/solvent phase ratio.

The uptake and release of solvents by the PMMA gels were measured gravimetrically, and the presence of unreacted monomer in solvents was assessed with Fourier Transform Infrared Spectroscopy (FITR). Small-angle $\mathrm{X}$-ray scattering (SAXS) measurements were then taken to detail the nanoscale structure and mesoporosity of the swollen organogels.

Finally, the organogels were applied on both model and real canvas samples that exhibited surface coatings representative of those commonly met in cleaning conservation interventions. The removal of the coatings and the absence of gel residues on the treated surfaces were checked with attenuated total reflectance Fourier transform infrared spectroscopy (ATR-FTIR).

\section{Experimental details}

\subsection{Chemicals}

MMA (Sigma-Aldrich, purity $\geq 99 \%$ ), ethylene glycol dimethylacrylate (EGDMA) (Sigma-Aldrich, purity 
$\geq 98 \%$ ), 2-butanone (MEK, Sigma-Aldrich, purity $99 \%$ ), ethyl acetate (Sigma-Aldrich, ACS reagents, purity $\geq 99.5 \%$ ), cyclohexanone (Fluka, purity $>98 \%$ ) and butyl acetate (Fluka, purity $>98.5 \%$ ) were used for the syntheses of the gels. To initiate the radical polymerization reaction, a lipophile starter was used, i.e., $\alpha, \alpha^{\prime}$-Azoisobutyronitrile (AIBN, Fluka, purity $98 \%$ ). $\mathrm{CaCO}_{3}$ and $\mathrm{Fe}_{2} \mathrm{O}_{3}$ were purchased from Fluka and used for the preparation of model canvas paintings (see also Sect. 2.3). All chemicals were used as received.

\subsection{Gel preparation and characterization}

The poly(methyl methacrylate) (PMMA) gels were synthesized in special PTFE beakers (with an inner diameter of $2 \mathrm{~cm}$ ) resistant to chemicals and high temperatures. The monomer MMA, solvent and cross-linker $(<1 \% \mathrm{w} / \mathrm{w})$ were added in the right proportions, and then, the mixture was bubbled with nitrogen in order to remove dissolved oxygen which acts as a radical inhibitor. Then, $\operatorname{AIBN}(<2 \%$ w/w of monomer weight) was added. The containers were sealed and placed into an oven at $65{ }^{\circ} \mathrm{C}$ for $5 \mathrm{~h}$. In order to remove unreacted monomers, the gels were washed by putting them into plastic vials $(30 \mathrm{ml})$ filled with the same solvent used for the synthesis. After $24 \mathrm{~h}$, the washing solvent was discarded. Four washing cycles were carried out after each synthesis. The presence of unreacted monomer in the washing solvent was assessed through ATR-FTIR, using a Thermo Nicolet Nexus 870 FTIR spectrometer. The spectra were obtained from 128 scans with $4 \mathrm{~cm}^{-1}$ of optical resolution, in the $4000-400 \mathrm{~cm}^{-1}$ range.

The polymerization reaction yield is defined as follows:

yield $=\frac{\text { amount of obtained polymer }}{\text { amount of monomers }} \times 100$

The "amount of obtained polymer" after the reaction is calculated by weighing the oven-dried gel (after the washing cycles), and the "amount of monomers" is the weight of monomer used for the preparation of the gel. For each gel, the yield was calculated as the average on two syntheses.

For the solvent uptake kinetics, the PMMA gels were heated up to $60^{\circ} \mathrm{C}$ from room temperature (heating rate $10{ }^{\circ} \mathrm{C} / \mathrm{min}$ ), kept at $60{ }^{\circ} \mathrm{C}$ for $1 \mathrm{~h}$ and finally heated up to $200{ }^{\circ} \mathrm{C}$ (heating rate $10^{\circ} \mathrm{C} / \mathrm{min}$ ) and weighted. The dried gels were then immersed in the corresponding solvent, and their weight was measured at given times (every $30 \mathrm{~min}$ the first day, and then once a day up to complete swelling). The percentage of solvent in the gels during solvent uptake tests was calculated as follows:

Solvent percentage $(\%)=\frac{W_{\mathrm{i}}-W_{\mathrm{d}}}{W_{\mathrm{d}}} \times 100$ where $W_{\mathrm{i}}$ is the weight of the gel at time $i$, and $W_{\mathrm{d}}$ is the weight of the dried gel. The equilibrium solvent content $(Q)$ was calculated using Eq. 2, where $W_{\mathrm{i}}$ is substituted with the weight of the completely swollen gel.

Small-angle X-ray scattering measurements were taken with a HECUS SWAXS camera (Kratky-type) equipped with a position-sensitive detector (OED $50 \mathrm{M}$ ) containing 1024 channels of width $54 \mu \mathrm{m}$. $\mathrm{Cu} \mathrm{K} \alpha$ radiation of wavelength $\lambda=1.542 \AA$ was provided by a Seifert ID-3003 $\mathrm{X}$-ray generator (sealed-tube type), operating at a maximum power of $2 \mathrm{~kW}$. A 10-mm-thick nickel filter was used to remove the $\mathrm{Cu} K \beta$ radiation. The sample-to-detector distance was $273 \mathrm{~mm}$. The volume between the sample and the detector was kept under vacuum during the measurements to minimize scattering from the air. The Kratky camera was calibrated in the small-angle region using silver behenate ( $d=58.38 \AA$ ) [17]. Scattering curves were obtained in the q-range between 0.01 and $0.54 \AA^{-1}$, assuming that $\mathrm{q}$ is the scattering vector, $q=4 \pi / \lambda \sin \theta$, and $2 \theta$ the scattering angle. Gel samples were placed into a 1-mm demountable cell having Kapton films as windows. The temperature was set to $25^{\circ} \mathrm{C}$ and was controlled by a Peltier element, with an accuracy of $0.1{ }^{\circ} \mathrm{C}$. All scattering curves were corrected for the empty cell contribution and for the scattering of the Kapton films, considering the relative transmission factor. SAXS curves were iteratively desmeared using the procedure reported by Lake [18].

\subsection{Easel painting samples}

Lined canvas models, canvas painting models and a real canvas painting sample were used to assess the performance of the gels. The type of surface coating for each sample is summarized in Table 1.

Model samples of varnished canvas paintings were realized applying a mixed "preparation-painted" layer (prepared with $100 \mathrm{ml}$ of rabbit skin glue $10 \%$ in water, 60 gr of $\mathrm{CaCO}_{3}$ and $40 \mathrm{~g}$ of red pigment $\mathrm{Fe}_{2} \mathrm{O}_{3}$ ) over natural linen canvas. A varnish layer was then applied on top of the dry preparation-painted layer (see Fig. 1). Three types of models were realized, each coated with a different type of varnish based, respectively, on Regalrez 1094, Paraloid B72 and Mastic resin. The varnishes were selected according to representativeness criteria, among natural and synthetic coatings typically used in classic and modern restoration practice.

Regalrez 1094 is a hydrocarbon low molecular weight resin, soluble in both aliphatic and aromatic solvents. The varnish is typically prepared as a $10 \%$ Regalrez 1094 solution in white spirit, adding $2 \%$ Kraton G1650 (a plasticizer styrene-ethylene-butylene-styrene SEBS copolymer) and $2 \%$ Tinuvin 292 (a hindered amine light 
Table 1 Samples of lined canvas and canvas paintings used for the cleaning tests

\begin{tabular}{lll}
\hline Canvas samples name & Description & Surface coating \\
\hline LM1 & Lined canvas model & Mowilith DM5 \\
LM2 & Lined canvas model & Plextol B500 \\
LM3 & Lined canvas model & Plexisol P550 \\
CM1 & Canvas painting model & Regalrez 1094 \\
CM2 & Canvas painting model & Paraloid B72 \\
CM3 & Canvas painting model & Mastic \\
"Peasant with chickens" & Nineteenth- to twentieth-century oil on canvas & Terpenic resin \\
\hline
\end{tabular}

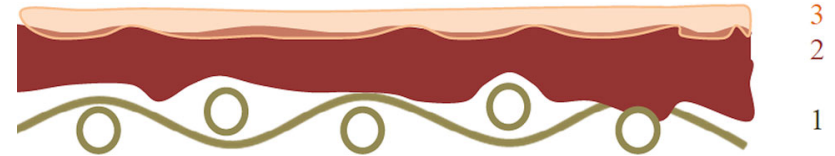

Fig. 1 Schematic cross section of a (varnished) model canvas painting (CM1-3, see Table 1) used for the assessment of gels. 1 Natural linen canvas. 2 Mixed "preparation-painted" layer (made of skin glue, $\mathrm{CaCO}_{3}$ and $\mathrm{Fe}_{2} \mathrm{O}_{3}$ red pigment). 3 Varnish layer

stabilizer, HALS) to inhibit photochemical degradation [19]. Paraloid B72 (Rohm \& Haas, USA) is a 70:30 ethyl methacrylate/methyl acrylate copolymer, applied in this case in diacetone alcohol solution (15\%). Mastic (a natural triterpenoid resin) was applied in turpentine solution $(20 \%)$.

Three types of lined canvas model samples were realized by applying different synthetic polymer products (i.e., Mowilith DM5, Plextol B500 and Plexisol P550, all commonly used as canvas adhesives) onto natural linen canvas. Mowilith DM5 is a copolymer of vinyl acetate (65\%) and $n$-butyl acrylate (35\%) in aqueous emulsion. The acrylate is used as an internal plasticizer. Plextol B500 is a copolymer of ethyl acrylate (60\%) and methyl methacrylate $(40 \%)$ in aqueous emulsion. Plexisol P550 is a solution of $n$-butyl methacrylate in hydrocarbons $(40 \% \mathrm{w} / \mathrm{w})$.

Finally, the gels were tested over a late nineteenth- to early twentieth-century oil on canvas painting ("Peasant with chickens") that exhibits an aged and yellowed terpenic varnish layer.

In all cases, the gels were applied directly onto the samples (application time 5-10 $\mathrm{min}$ ) to remove the surface coatings. The varnishes and adhesives were either solubilized (and migrated into the gels) or swollen, and then removed with a gentle mechanical action using a cotton swab.

The presence of gel residues over the surface was checked with ATR-FTIR performed on the canvas samples after the application and removal of the gels. A Thermo Nicolet Nexus 870 FTIR spectrometer equipped with a Golden Gate diamond cell was used. Data were collected with an MCT detector with a sampling area of $150 \mu \mathrm{m}^{2}$.
The spectra were obtained from 128 scans with $4 \mathrm{~cm}^{-1}$ of optical resolution, in the $4000-650 \mathrm{~cm}^{-1}$ range.

\section{Results and discussion}

The PMMA gels were prepared as cylinders with a diameter of $2 \mathrm{~cm}$ and height ranging from $2-3 \mathrm{~mm}$ to ca. $2 \mathrm{~cm}$ (see Fig. 2). In general, all the synthesized gels appear as gummy and transparent, with good mechanical stability to handling and manipulation.

The yield of the polymerization reaction varied with the type of solvent and the amount of starter (AIBN) used. The MMA-MEK gel was obtained using $0.25 \%$ AIBN (w/w of monomer weight), with a yield of $43 \%$. The MMA-Cyclo gel was also obtained using $0.25 \%$ AIBN; however, it was not possible to calculate the yield because the high boiling point of cyclohexanone did not allow obtaining a perfectly dried gel.

The MMA-EA and MMA-BA systems were obtained raising the amount of AIBN up to ca. $1.6 \%$, and these two
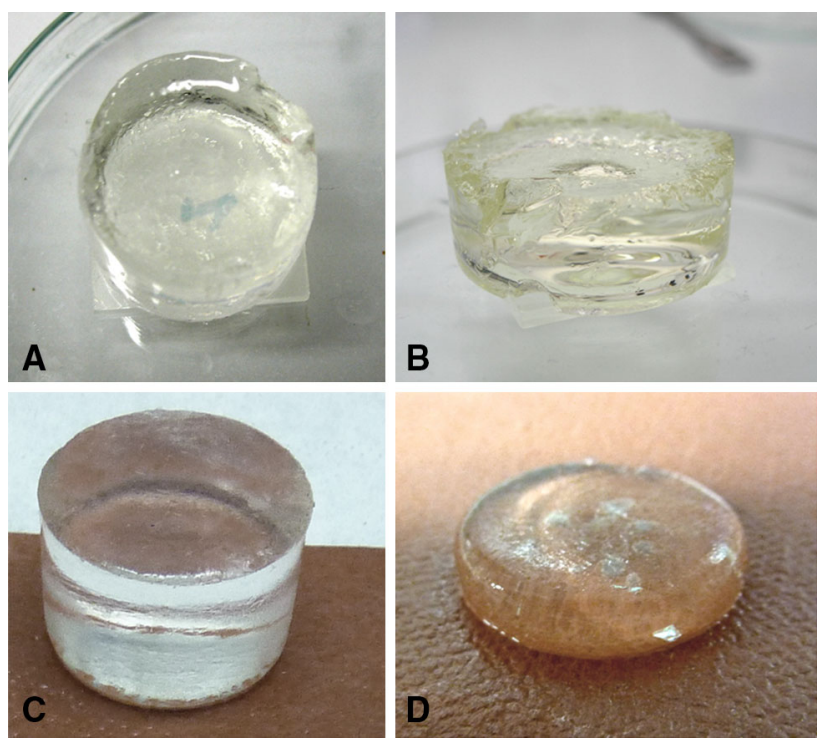

Fig. 2 pMMA gels synthesized in different solvents. a MMA-MEK; b MMA-Cyclo; c MMA-EA; d MMA-BA 
systems showed the highest polymerization yield $(83 \%)$. An increase in cross-linking between the polymer chains can result in a decrease in the mesh size, i.e., the dimension of the nanosized pores of the polymer network. However, the main factor influencing the network's porosity is the equilibrium solvent content $\mathrm{Q}$ (the higher the value of $\mathrm{Q}$, the higher the porosity), which depends also on the polymer-solvent affinity.

In all cases, the polymerization of MMA is not complete, and right after the syntheses, the PMMA organogels contain significant amounts of unreacted monomers. Therefore, washing cycles were carried out on gels to remove the monomers. The washed gels were then soaked in $5 \mathrm{ml}$ of the corresponding solvent and let exchange overnight. The solvent was then removed and analyzed using ATR-FTIR, comparing the obtained spectrum with that of the pure solvent, of a $1 \% \mathrm{w} / \mathrm{w}$ solution of MMA in the solvent, and of pure MMA (see Figs. 3, 4).

After two complete washing cycles, the spectrum of the solvent that exchanged overnight with the washed gel shows absorption bands whose intensity is comprised between those of the pure solvent and the $1 \%$ MMA solution. This means that after two washing cycles, the amount of unreacted monomer in the gels is less than $1 \%$. Overall, four washings are necessary to obtain a monomer free gel.

All systems, if left inside the corresponding solvent, tend to adsorb liquid and significantly swell.

The drying and re-swelling of the gels were carried out as described in Sect. 2.2, in order to study the affinity between the different solvents and the PMMA polymer network, and to investigate the transport mechanism of the solvent molecules within the network.

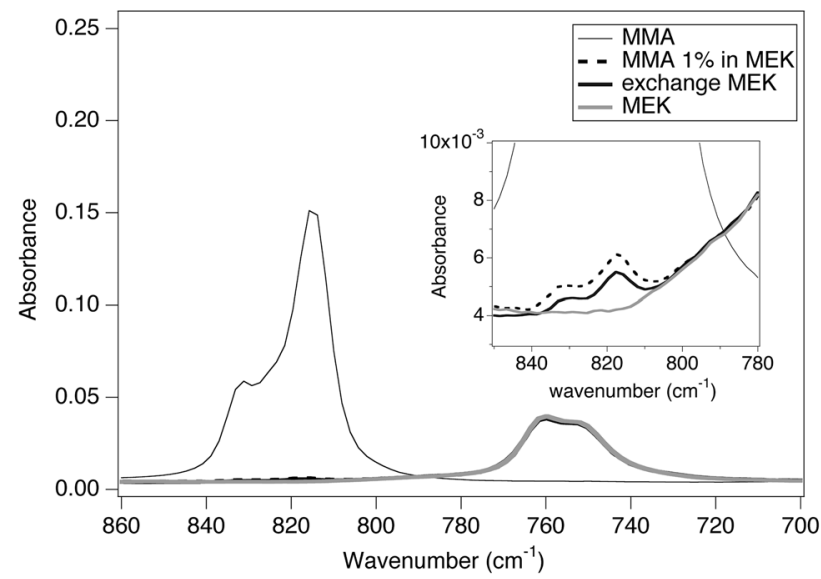

Fig. 3 ATR-FTIR spectrum of MEK that was let to exchange with the MMA-MEK organogel after two washing cycles ("exchange MEK"), as compared to the spectra of pure MEK, MMA, and of a $1 \%$ MMA solution in MEK

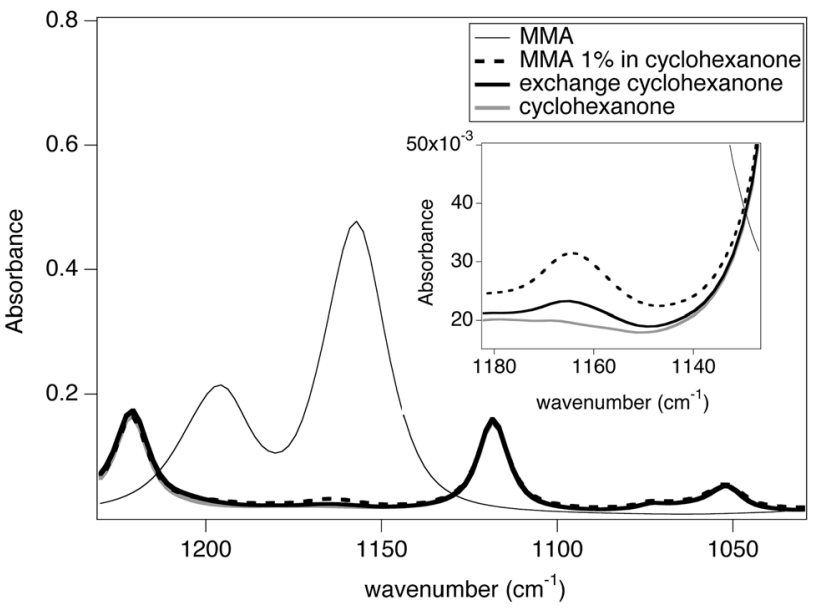

Fig. 4 ATR-FTIR spectrum of cyclohexanone that was let to exchange with the MMA-Cyclo organogel after two washing cycles ("exchange cyclohexanone"), as compared to the spectra of pure cyclohexanone, MMA, and of a $1 \%$ MMA solution in cyclohexanone

The equilibrium solvent content $(Q)$ was calculated both for gels that were let equilibrate with the solvent for 7 days right after the preparation, and for gels that were dried after preparation, and then swollen with the solvents. The two different values of $Q$ were defined as $Q_{1}$ and $Q_{2}$. The highest values of both $Q_{1}$ and $Q_{2}$ were found for the MMAMEK system, suggesting that MEK has the highest affinity for the PMMA network as compared to the other solvents in the set. The MMA-Cyclo and MM-EA systems show very similar $Q$ values, while the MMA-BA system exhibited the lowest equilibrium solvent content.

Moreover, it was found that $Q_{1}>Q_{2}$ (see Table 2), and the difference was attributed to the irreversible collapse of gel pores during the drying step. For MMA-EA, the slightly higher value of $Q_{2}$ might be explained considering that cracks form upon drying, increasing the macroporosity of the gel. In all cases, the percentage of liquid phase used during the preparation is well below the maximum percentage reached at the equilibrium.

Table 2 Equilibrium solvent content for gels that were let equilibrate with the solvent for 7 days right after the preparation $\left(Q_{1}\right)$, and for gels that were dried after preparation, and then swollen with the solvents $\left(Q_{2}\right)$

\begin{tabular}{llll}
\hline Gel & Preparation liquid $(\%)$ & $Q_{1}(\%, \pm 0.1)$ & $Q_{2}(\%, \pm 0.1)$ \\
\hline MMA-MEK & 59.5 & 91.1 & 90.6 \\
MMA- & 59.5 & 76.8 & 75.8 \\
Cyclo & & & \\
MMA-EA & 59.5 & 77.8 & 79.1 \\
MMA-BA & 59.5 & 64.5 & 62.9 \\
\hline
\end{tabular}

The liquid content used for preparing the gels is shown for comparison 

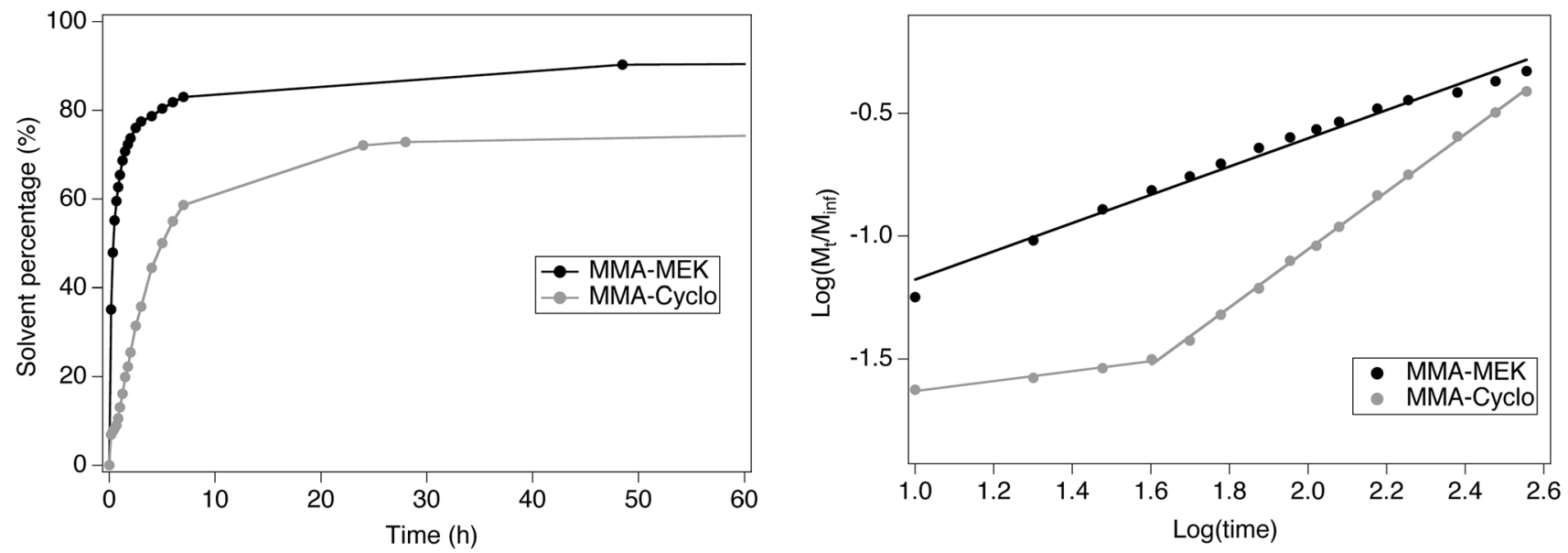

Fig. 5 (Left Panel) Plot of the solvent uptake (\%) versus time for dried MMA-MEK and MMA-Cyclo gels. The lines are simply guides. (Right panel) Plot of $\log \left(M_{\mathrm{t}} / M_{\mathrm{inf}}\right)$ versus $\log (t)$ and linear fitting $\left(R^{2}>0.98\right)$ for the linear part of the uptake curves

Figure 5 (left panel) shows the solvent uptake of the dried MMA-MEK and MMA-Cyclo organogels plotted versus time. By investigating the transport mechanism of the solvent molecules into the gel matrix, it is possible to obtain more information on the influence of solvents in the gelling process and in the final structural properties of the gels. The uptake process can be treated as a flux of solvent molecules moving from a region at high concentration (bulk liquid phase) to a region at low concentration (the gel matrix) [20]. For the first part of the solvent uptake curve, i.e., when the ratio between the liquid uptake at time $t\left(M_{\mathrm{t}}\right)$ and at equilibrium $\left(M_{\mathrm{inf}}\right)$ is less than 0.6 , the following semiempirical equation can be used to describe the process:

$\frac{M_{\mathrm{t}}}{M_{\mathrm{inf}}}=k t^{n}$

where $k$ is a constant that includes the chemical, structural and geometric characteristics of the sample, and the exponent $n$ defines the mechanism of solvent transport in the gel [21]. This power law considers the dynamic swelling of polymers as a superposition of two transport mechanisms, i.e., diffusion governed by Fick's law (often referred to as "Fickian diffusion"), and a "Case II transport" mechanism where the relaxation of the polymer macromolecules upon solvent imbibition is the rate-controlling step.

For cylinder geometry (such as the PMMA organogels that we prepared), the value of $n$ is related to the transport mechanism as follows: $n<0.45$ indicates that the diffusion of solvent is governed by Fick's law; $0.45<n<0.89$ indicates "anomalous transport" (a combination of diffusion governed by Fick's law and "Case II transport"); $n=0.89$ indicates that a pure "Case II transport" mechanism is operating (i.e., relaxation controlled kinetics) [22, 23].

Diffusion governed by Fick's law applies when the absorbed solvent does not interact with the porous gel substrate, and the diffusion is only governed by the solvent concentration gradient. Otherwise, either anomalous transport or Case II transport takes into account the influence of polymer relaxation on the movement of the solvent molecules in the matrix.

As shown in Fig. 5 (left panel), the MMA-MEK gel shows the highest percentage of liquid uptake. When $\log \left(M_{\mathrm{t}} / M_{\text {inf }}\right)$ is plotted versus $\log (t)$, the linear regression fittings of the first part of solvent uptake curves allows obtaining the value of $n$. The linear regressions fit quite well the solvent uptake data, with $R^{2}$ always greater than 0.98. For the MMA-MEK gel, $n=0.65$, which highlights an "anomalous transport" mechanism that indicates high affinity between the polymer and the solvent. On the contrary, in the case of MMA-Cyclo, two different linear trends are observed: a first part characterized by a lower uptake rate (where $n=0.20$ ) and a second part with a significant higher rate $(n=1.07)$. This indicates that, in the case of the gel system containing cyclohexanone, a structure collapse could occur upon drying, which confirms the fundamental role of solvent as porogenic agent in the gelling process.

Moreover, the relatively low volatility of the loaded solvents made it difficult to obtain xerogels through lyophilization without significantly affect the pore structure; therefore, the investigation of the PMMA organogels through scanning electron microscopy did not provide exhaustive information on their porosity. Thus, the mesoporosity and the nanoscale structure of the gels were determined through SAXS analysis directly on the swollen organogels, thanks to the presence of a good scattering length density contrast between all the gel phases. Figure 6 shows the obtained SAXS curves for MMA-MEK and MMA-Cyclo after subtraction of the empty cell and Kapton contribution. In order to extract the structural information, 

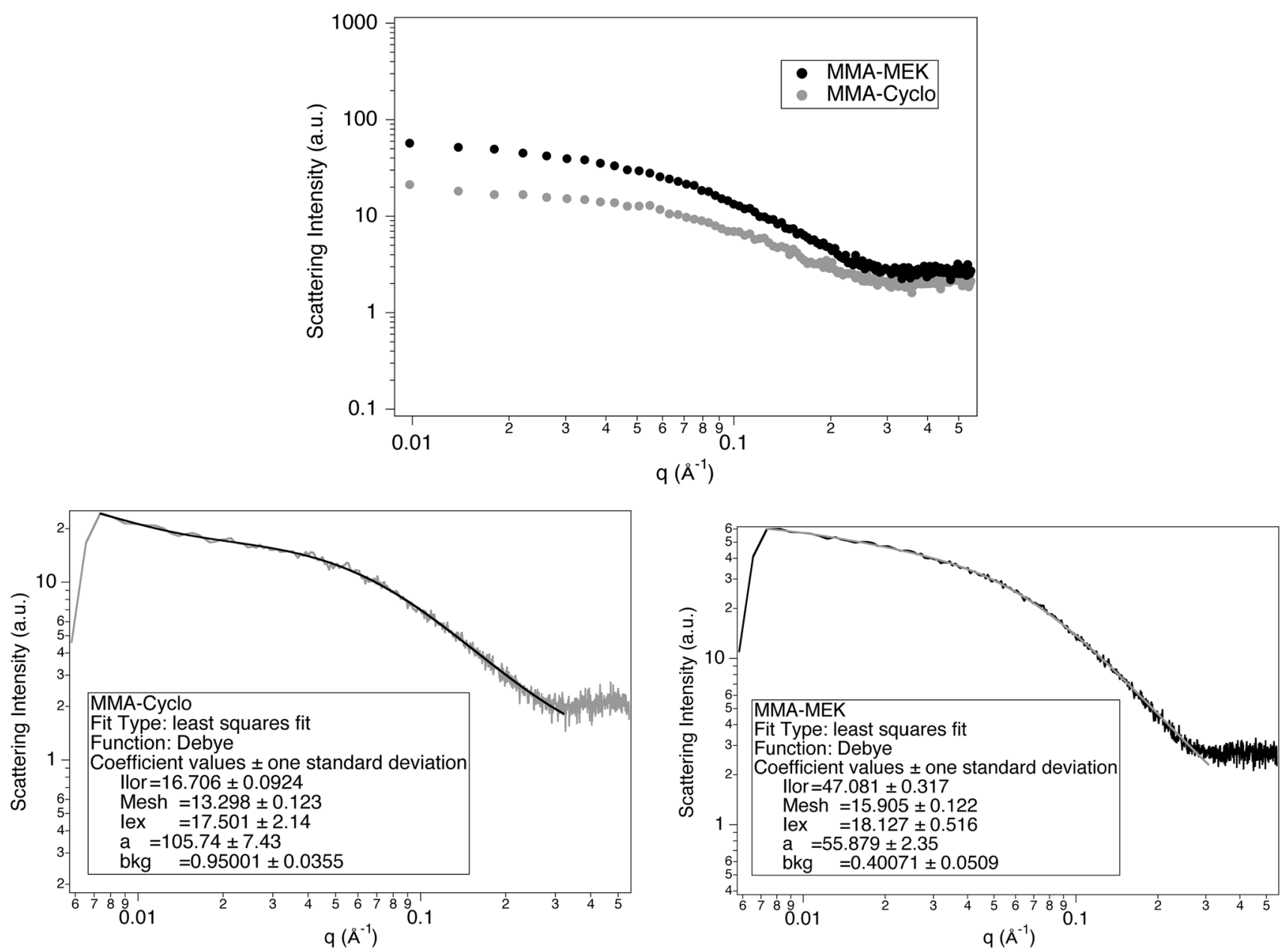

Fig. 6 (Top Panel) SAXS curves of the swollen MMA-MEK and MMA-Cyclo gels. (Bottom panels) SAXS data and Debye-Bueche fitting for the MMA-Cyclo (left) and the MMA-MEK (right) swollen gels

the SAXS curves were fitted using the Debye-Bueche approach [24], where the SAXS intensity distribution is split in two main contributions and an instrumental flat background:

$I(q)=I_{\mathrm{Lor}}(q)+I_{\mathrm{ex}}(q)+b k g$

The $I_{\text {Lor }}(q)$ Lorentzian term accounts for the scattering associated with a tridimensional network with a characteristic mesh size and can be expressed as:

$I_{\text {Lor }}(q)=\frac{I_{\text {Lor }}(0)}{1+q^{2} \zeta^{2}}$

where $I_{\text {Lor }}(0)$ is the Lorentzian intensity at $q=0$ and $\zeta$ is the average mesh size of the network, which is associated with the mesoporosity of the gel structure.

The second term is the excess scattering, which is introduced to account for the low- $q$ scattering due to inhomogeneities in the structure, such as solid-like polymer domains:
$I_{\mathrm{ex}}(q)=\frac{I_{\mathrm{ex}}(0)}{\left(1+q^{2} a^{2}\right)^{2}}$

where $I_{\mathrm{ex}}(0)$ is the excess intensity at $q=0$ and $a$ is the average dimension of the inhomogeneity domains accessible by the SAXS experiment. It is also interesting to

Table 3 SAXS fitting parameters of the MMA-MEK and MMACyclo gels

\begin{tabular}{lcc}
\hline SAXS parameters & Gel \\
\cline { 2 - 3 } & MMA-MEK & MMA-Cyclo \\
\hline$I_{\text {Lor }}(0)$ & $47.1 \pm 0.3$ & $16.7 \pm 0.1$ \\
$\zeta(\mathrm{nm})$ & $1.59 \pm 0.01$ & $1.33 \pm 0.01$ \\
$I_{\mathrm{ex}}(0)$ & $18.1 \pm 0.5$ & $17 \pm 2$ \\
$a(\mathrm{~nm})$ & $5.5 \pm 0.2$ & $10.5 \pm 0.7$ \\
$b k g$ & $0.40 \pm 0.05$ & $0.95 \pm 0.04$ \\
$I_{\text {ex }}(0) / I_{\text {Lor }}(0)$ & $0.38 \pm 0.01$ & $1.0 \pm 0.1$ \\
\hline
\end{tabular}



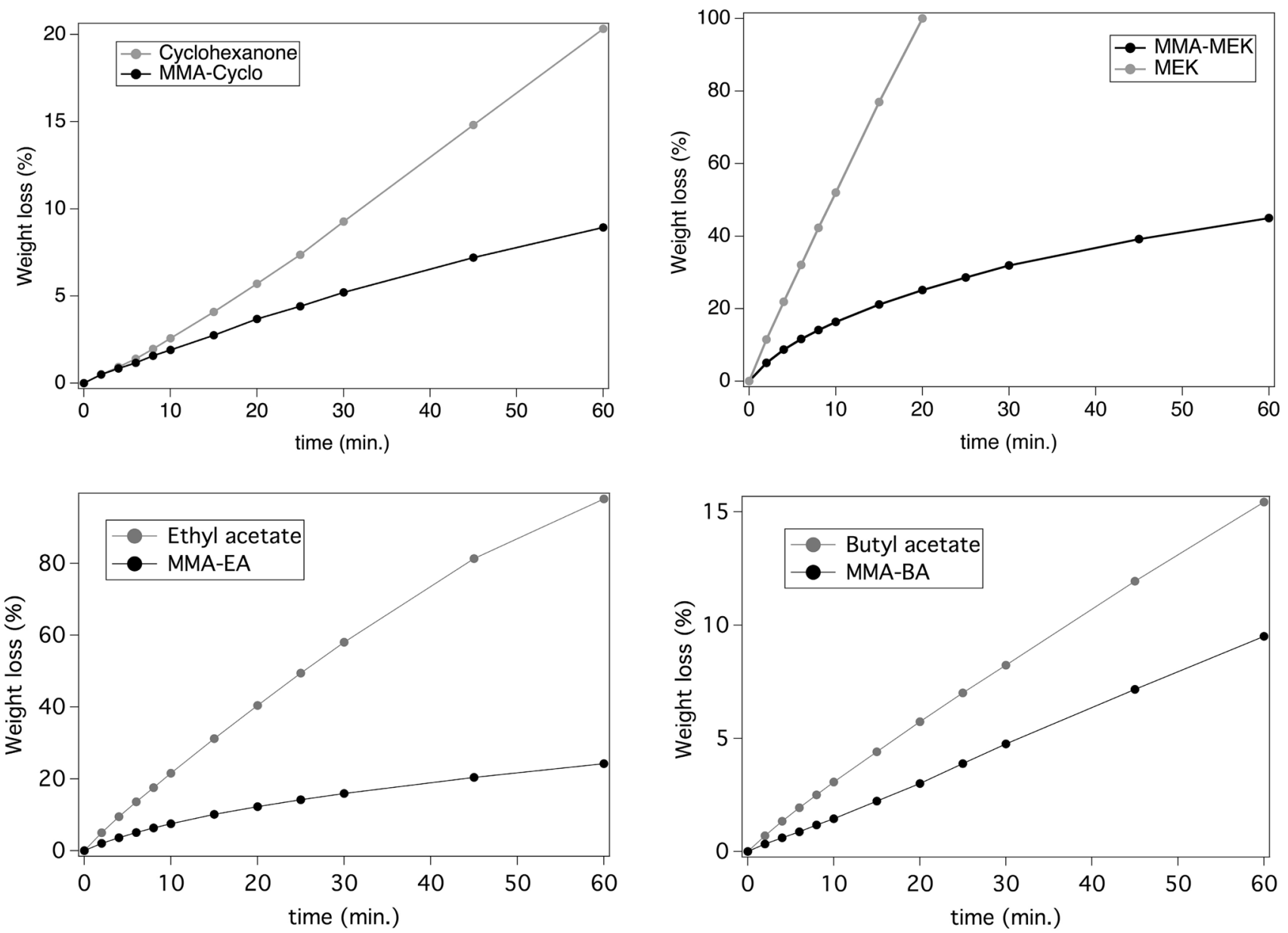

Fig. 7 Weight loss through evaporation of free solvents (from petri dishes) as compared to that of solvents confined in the PMMA gels

consider the $I_{\mathrm{ex}}(0) / I_{\mathrm{Lor}}(0)$ ratio, which is proportional to inhomogeneity/mesh volume fractions.

The results of the Debye-Bueche fitting are shown in Fig. 6, and the obtained parameters are resumed in Table 3.

Both MMA-MEK and MMA-Cyclo gels are prepared using the same monomer concentration and monomer/ cross-linker ratio. Only the liquid phase is changed. Thus, the SAXS parameters can be interpreted taking into account the different solvent uptake data presented before. The system that absorbs more solvent is MMA-MEK, which also shows a higher mesh size and a lower inhomogeneity domain size than MMA-Cyclo. In fact, the mesh size is known to depend mainly on the solvent equilibrium content [25], i.e., larger quantities of absorbed solvent require a larger pores volume, and thus, also the mesopores become larger. Regarding the inhomogeneity dimension, for a clearer discussion of the obtained data, it is useful to recall the classification of inhomogeneities (which are inevitably formed during the cross-linking of the polymer chains in the solvent) as spatial, topological and connectivity inhomogeneities [26]. The spatial inhomogeneities are due to nonuniform spatial distributions of cross-links and are mainly studied through SAXS or small-angle neutron scattering (SANS) techniques. The topological inhomogeneities are due to the presence of loops, trapped entanglements and dangling chains. The connectivity inhomogeneities are related to the size and distributions of clusters. Inhomogeneities increase with the number of cross-links and concentration of polymer in the gel network [26-28]. The $a$ values obtained here for the MMA gels confirm this behavior.

Besides gels' solvent uptake and mesoporosity, we also investigated the ability of the PMMA gels to reduce the evaporation of the confined solvents.

Both free and confined solvents were exposed at room temperature $\left(25^{\circ} \mathrm{C}\right)$ and RH $60 \%$. The loss of weight of swollen PMMA gels was compared to that of petri dishes containing the same mass of free solvents. In this case, gel cylinders with a height of $1.2 \mathrm{~cm}$ and a base diameter of $2 \mathrm{~cm}$ were used. The size of the petri dishes (diameter $=10.9 \mathrm{~cm}$ ) was selected to obtain a homogeneous film of free solvent (i.e., leaving no gaps) while maximizing the 
spreading of the solvent and the surface area exposed to air. The aim was to simulate the spreading of solvents when they are used as nonconfined in cleaning operations, as
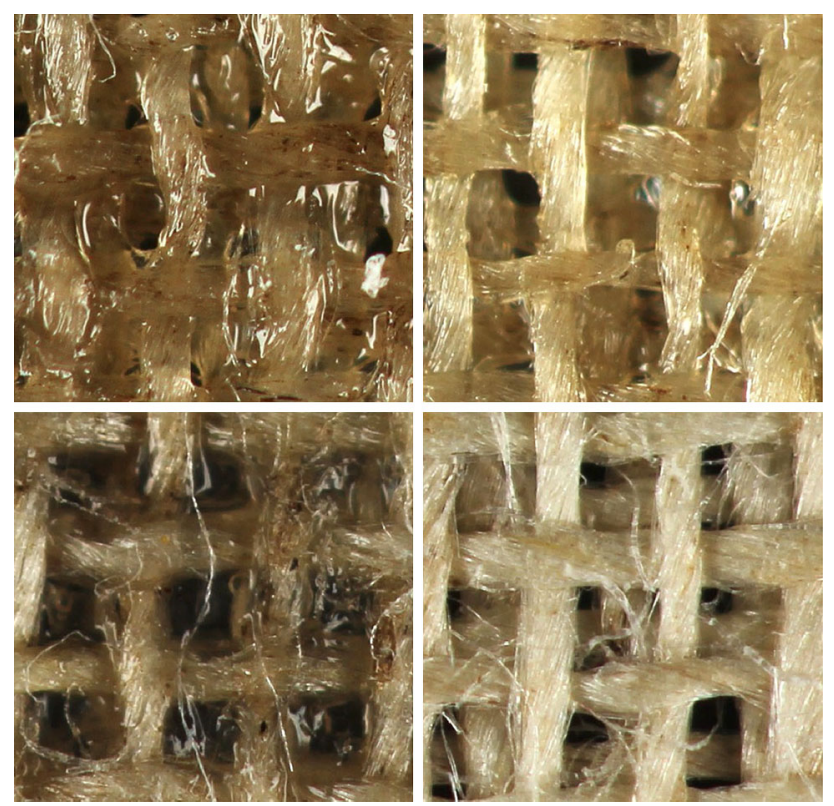

Fig. 8 Macropictures of lined canvas models. (Top row) Model LM1 before (left) and after (right) the removal of Mowilith DM5 using a MMA-EA organogel. (Bottom row) Model LM2 before (left) and after (right) the removal of Plextol B500 using a MMA-BA organogel
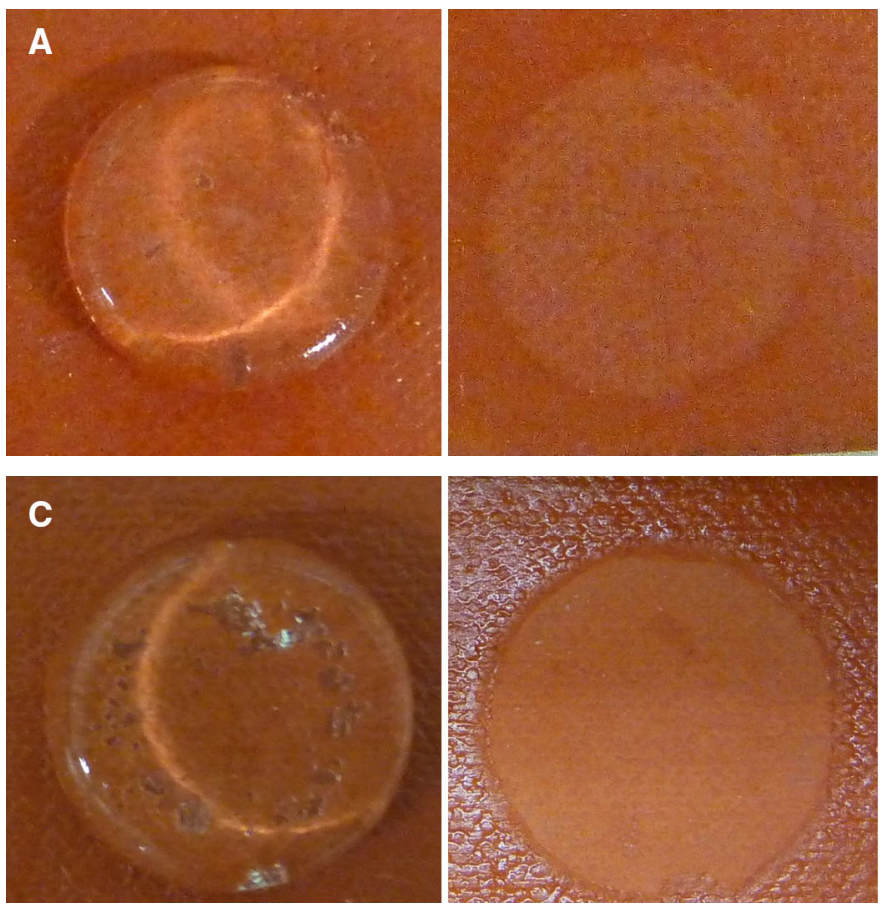

Fig. 9 Removal of surface coatings from canvas painting models using PMMA gels. a Removal of Paraloid B72 from model CM2 using a MMA-EA gel (normal light); $\mathbf{b}$ removal of Paraloid B72 from model CM2 using a MMA-BA gel (grazing light); c removal of opposed to solvents confined in the PMMA organogels. In this case, the gels and free solvents were not placed on porous surfaces as we wanted to investigate the evaporation of solvents as separated from the influence of capillary suction by the wet surface.

The gels, or the petri dishes, were placed on the pan of an analytical balance. The side glass doors of the balance enclosure were shut, while the top door was left open to allow air circulation as the weight loss was being recorded. Only normal room (laboratory) air circulation was used (no ventilation, no aspiration).

Results are shown in Fig. 7. The evaporation kinetics was interrupted after an hour because the application time of organogels is typically shorter. The measured evaporation rates of the free solvents are consistent with their different volatilities, with MEK and EA showing a rate significantly higher than that of BA and Cyclo. When the solvents are confined in the gels, it is evident that the evaporation rate is reduced due to the retention power of the polymer network. This is an important applicative feature, because lower evaporation rates decrease the impact of solvents on operators. The rate of the confined solvents is still consistent with their volatilities, and the different shapes of the weight losses are to be ascribed also to other factors, such as the structural properties of the gels, the gel-solvent affinity and the solvent transport mechanism within the gel.
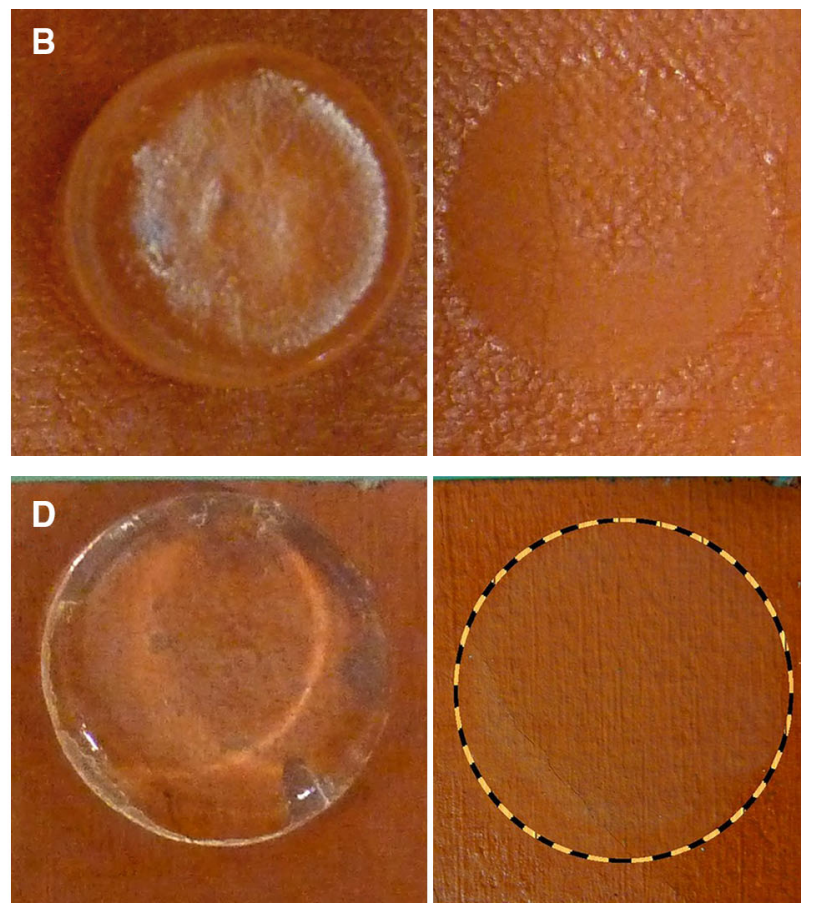

mastic from model CM3 using a MM-EA gel (grazing light); d removal of Regalrez 1094 from model CM1 using a MMA-BA gel (normal light); the dotted line encircles the cleaned area 
The organogels were then tested on models of lined canvas and canvas paintings.

For what concerns the removal of lining adhesives from models, the best results were obtained using MMA-EA for the removal of Mowilith DM5, and MMA-BA or MMAMEK for the removal of Plextol B500 (see Fig. 8).
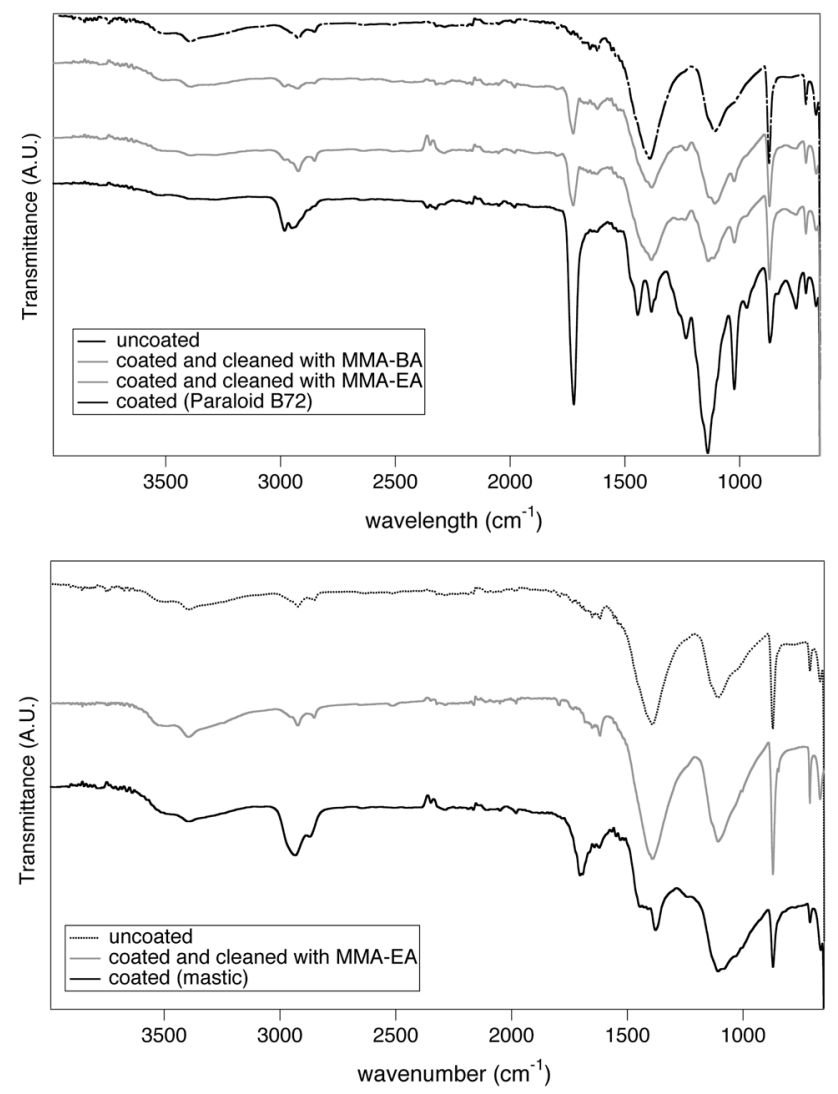

Fig. 10 ATR-FTIR spectra of canvas painting models (CM2, CM3) with no surface coating, coated with natural (mastic) or synthetic (Paraloid B72) coatings, and cleaned using the PMMA gels

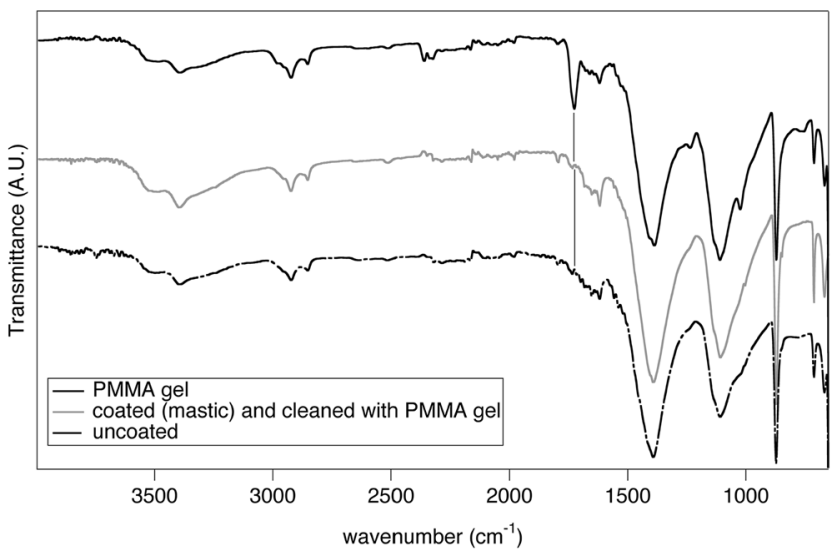

Fig. 11 (Left) ATR-FTIR spectra of a PMMA gel lying on a canvas painting model ("PMMA gel"), of a canvas painting model with no surface coating, and of a canvas painting model coated with mastic (CM3)
For the removal of varnishes from the canvas painting models, only the gels containing ethyl acetate and butyl acetate were tested, because these solvents match the swelling areas of the selected varnishes (as defined by Teas solubility parameters) much better than cyclohexanone and methyl ethyl ketone. The best results were obtained using MMA-EA and MMA-BA for the removal of Paraloid B72, and MMA-EA for the removal of mastic (see Fig. 9).

Figure 10 shows the ATR-FTIR spectra of the canvas painting models before and after the application of the PMMA gels, as compared to models that were not coated with varnishes. The strong decrease in the characteristic carbonyl absorption peaks of Paraloid B72 $\left(1725 \mathrm{~cm}^{-1}\right)$ and mastic $\left(1705 \mathrm{~cm}^{-1}\right)$ indicates the removal of a large portion of the varnishes. Application time of 5-10 min was used to thin the varnishes, as frequently required in real conservation interventions. However, it is possible to extend the application time (e.g., up to $20 \mathrm{~min}$ or more) in order to completely remove the coating.

In the spectrum of the treated canvas painting model (i.e., after application and removal of the PMMA gel), the characteristic carbonyl band of PMMA $\left(1726 \mathrm{~cm}^{-1}\right)$ is not observable, confirming that no residues of gel are present on the surface (see Fig. 11). This feature is particularly advantageous for practical applications, because it avoids the need of any rinsing step following the cleaning intervention, which might be problematic for mechanically weak or solvent-sensitive surfaces. In fact, traditional thickeners and "gel-like" systems based on cellulose ethers or polyacrylic acid (i.e., "physical" systems, where the network is made of primary bonds) are prone to leaving residues of gellant and nonvolatile components [8, 10], and in some cases (cellulose ethers), the residues are hard to remove even when rinsing is carried out [5].

Finally, the application of a PMMA gel (MMA-EA) on a real canvas painting ("Peasant with chickens") is shown in

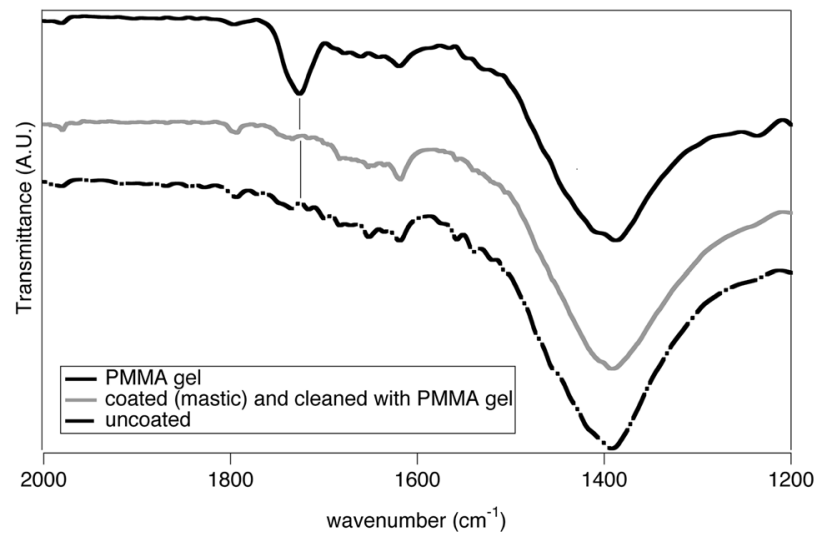

and treated using the PMMA gel. (Right) Detail of the $2000-1200 \mathrm{~cm}^{-1}$ spectral region, showing the absence of the carbonyl band of PMMA $\left(1726 \mathrm{~cm}^{-1}\right)$ in the spectrum of the model treated with the gel 
Fig. 12 Application of a MMA-EA gel onto a canvas painting (sample "Peasant with chickens," nineteenth- to twentieth-century oil on canvas). a Panel showing the aged, strongly yellowed terpenic varnish coating the painted layer. b Application of the MMA-EA gel onto the painting surface. c The application spot right after the removal of the gel, showing the swollen and softened varnish layer.

d Application area after the mechanical removal of the swollen varnish. e The MMAEA gel after the application
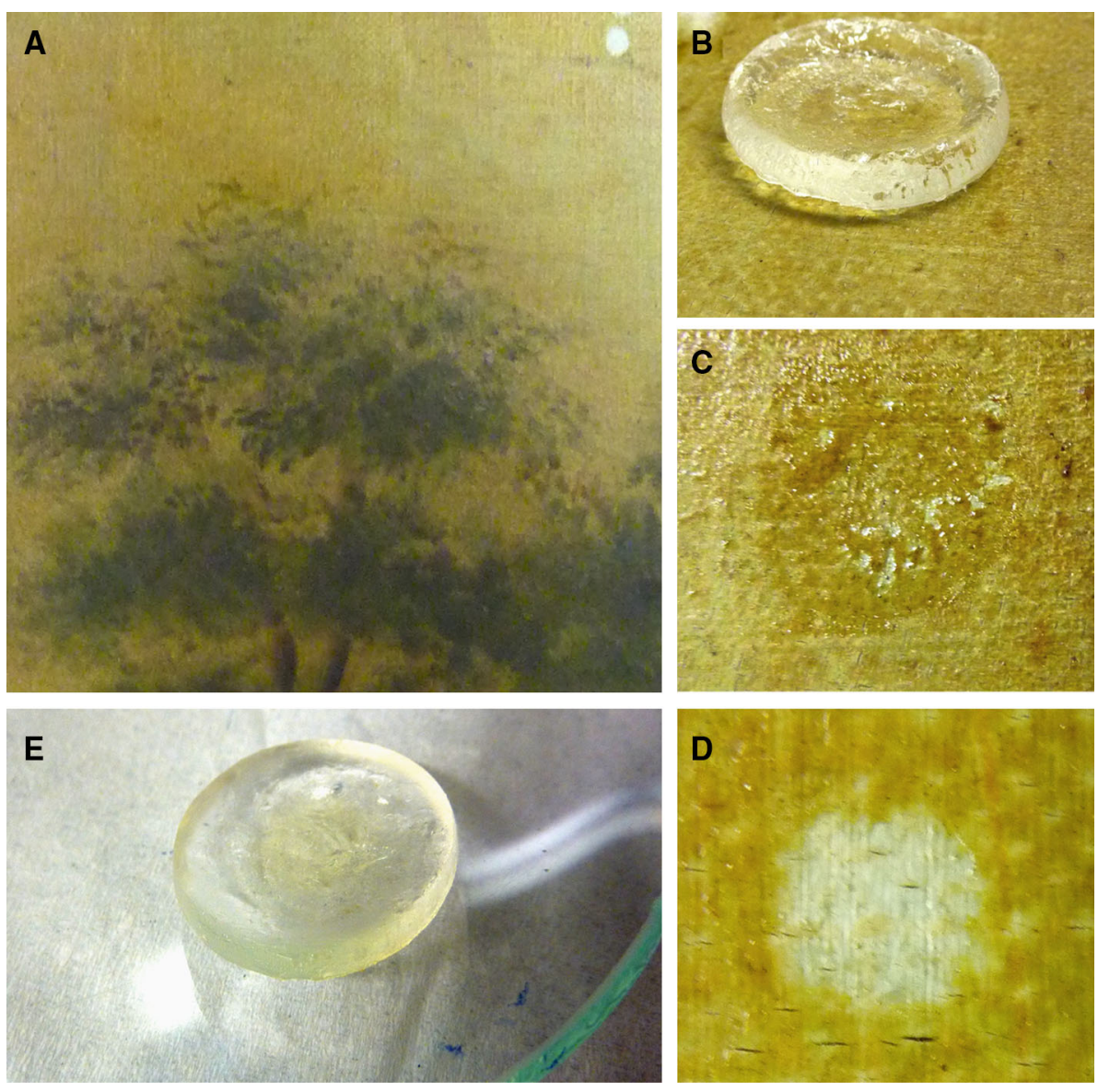

Fig. 12. After $5 \mathrm{~min}$, the gel is removed from the surface, leaving a swollen and softened varnish layer that can be easily removed mechanically using a cotton swab. Moreover, the varnish is also partially solubilized and migrates into the gel, which yellows owing to the varnish uptake (see Fig. 12e).

\section{Conclusions}

Poly(methyl methacrylate) chemical organogels have been prepared using different solvents and applied for the first time for the removal of unwanted adhesives and varnishes from lined canvas and canvas painting samples. Both the uptake/release behavior and the mesoporosity of the gels were investigated. The SAXS analysis has shown a greater mesh size and smaller inhomogeneity domain size for the gel systems with a higher equilibrium solvent content. Solvent release kinetics have highlighted the capability of the gels of trapping the solvent inside their structure and, consequently, of reducing the solvent evaporation and the related health risk for operators (restorers, curators).
Applicative tests have provided good results: the gel systems enable the controlled swelling and softening of the selected natural and synthetic coatings. The coatings are either solubilized (and migrate into the gels) or swollen and softened, and then removed with a light mechanical action. Changing the solvent used for the gel preparation allows targeting the removal of different coatings such as vinyl acetate and acrylate copolymers or natural terpenic resins.

Moreover, the PMMA organogels exhibit good optical transparency, which is advantageous for applications on artifacts since the treated surface can be directly observed during the cleaning operation. ATR-IR analysis confirmed that no gel residues are left on the samples' surface after the treatment with the gels.

Overall, PMMA chemical organogels may represent a real alternative to the widely used physical "gel-like" networks and thickeners for confining cleaning solvents.

Acknowledgments CSGI, MIUR and European Union (Project NANOFORART, FP7-ENV-NMP-2011/282816) are acknowledged for financial support. 


\section{References}

1. P. Dietemann, C. Higitt, M. Kälin, M.J. Edelmann, R. Knochenmuss, R. Zenobi, J. Cult. Herit. 10, 30 (2009)

2. R. Howells, A. Burnstock, G. Hedley, S. Hackney, in Adhesives and Consolidants, ed. by N.S. Brommelle, E.M. Pye, P. Smith, G. Thomson (IIC, London, 1984)

3. J.L. Down, M.A. MacDonald, J. Tétreault, R. Scott Williams, Stud. Conserv. 41(1), 19 (1996)

4. D. Chelazzi, A. Chevalier, G. Pizzorusso, R. Giorgi, M. Menu, P. Baglioni, Polym. Degrad. Stab. 107, 314 (2014)

5. A. Casoli, Z. Di Diego, C. Isca, Environ. Sci. Pollut. Res. 21(23), 13252 (2014)

6. P. Baglioni, D. Chelazzi, R. Giorgi, Nanotechnologies in the Conservation of Cultural Heritage (Springer, Dordrecht, 2015), pp. 98-99

7. E. Carretti, S. Grassi, M. Cossalter, I. Natali, G. Caminati, R.G. Weiss, P. Baglioni, L. Dei, Langmuir 25, 8656 (2009)

8. A. Burnstock, T. Kieslish, in Proceedings of ICOM Committee for Conservation, 11th Triennal Meeting in Edimburgh, Scotland (James \& James, London, Sept. 1-6, 1996) p. 253

9. A. Burnstock, R. White, in Tradition and Innovation: Advances in Conservation, Contributions to the Melbourne Congress, 10-14 Oct 2000 (IIC), p. 38

10. D. Stulik, D. Miller, H. Khanjian, N. Khandekar, R. Wolbers, J. Carlson, W. Christian Petersen, in Solvent Gels for the Cleaning of Works of Art: The Residue Question, ed. by V. Dorge (Getty Publications, Los Angeles, 2004)
11. G. Pizzorusso, E. Fratini, J. Eiblmeier, R. Giorgi, D. Chelazzi, A. Chevalier, P. Baglioni, Langmuir 28, 3952 (2012)

12. J. Domingues, N. Bonelli, R. Giorgi, E. Fratini, F. Gorel, P. Baglioni, Langmuir 29, 2746 (2013)

13. J. Domingues, N. Bonelli, R. Giorgi, P. Baglioni, Appl. Phys. A 114, 705 (2014)

14. S. Pajevic, R. Bansil, C. Konak, Macromolecules 26, 305 (1993)

15. Ö. Pekcan, D. Kaya, M. Erdogan, Polymer 41, 4915 (2000)

16. H.J. Naghash, J. Appl. Polym. Sci. 116, 2465 (2010)

17. T. Blanton, T. Huang, H. Toraya, C.R. Hubbard, S.B. Robie, D. Louer, H.E. Gobel, G. Will, R. Gilles, T. Raftery, Powder Diffr. 10, 91 (1995)

18. J.A. Lake, Acta Crystallogr. 23, 191 (1967)

19. J. Whitten, AIC postprints, American Institute for Conservation 23rd Annual Meeting, St. Paul, Minn. AIC, Washington DC, p. 124

20. P.L. Ritger, N.A. Peppas, J. Control. Release 5, 37 (1987)

21. J. Siepmann, N.A. Peppas, Adv. Drug Deliv. Rev. 48, 139 (2001)

22. K. Kosmidis, E. Rinaki, P. Argyrakis, P. Macheras, Int. J. Pharm. 254, 183 (2003)

23. T. Alfrey, E.F. Gurnee, W.G. Lloyd, J. Polym. Sci. Part C Polym. Symp. 12, 249 (1966)

24. P. Debye, A.M. Beuche, J. Appl. Phys. 20, 518 (1949)

25. T. Canal, N.A. Peppas, J. Biomed. Mater. Res. A 23, 1183 (1989)

26. F. Ikkai, M. Shibayama, J. Polym. Sci. Part B Polym. Phys. 43, 617 (2005)

27. S. Panyukov, Y. Rabin, Phys. Rep. 269, 1 (1996)

28. L. Benguigui, F. Boue, EPJB 11, 439 (1999) 